\title{
Clinical Relevance of CD-68 Positive Cells in Normal Buccal Mucosa in Patients with Inflammatory Bowel Disease ${ }^{\dagger}$
}

\author{
Brankica Mijandrušić Sinčić, ${ }^{a, *}$ Maja Ilić Tomaš, ${ }^{\mathrm{b}}$ Margita Belušić Gobić, ${ }^{\mathrm{c}}$ Mirna Juretić, ${ }^{\mathrm{c}}$ \\ Dražen Kovač, ${ }^{\mathrm{d}}$ Andrica Lekić, ${ }^{\mathrm{e}}$ Ester Pernjak Pugel, ${ }^{\mathrm{f}}$ Robert Cerović, ${ }^{\mathrm{c}}$ and Davor Štimac ${ }^{\mathrm{a}}$ \\ ${ }^{a}$ Department of Gastroenterology, University Hospital Centre Rijeka, Rijeka, Croatia \\ ${ }^{\mathrm{b}}$ Department of Physiology and Immunology, Medical Faculty, University of Rijeka, Rijeka, Croatia \\ ${ }^{\mathrm{c}}$ Depertment of Maxillofacial and Oral Surgery, University Hospital Centre Rijeka, Rijeka, Croatia \\ ${ }^{\mathrm{d}}$ Department of Pathology, Medical Faculty, University of Rijeka, Rijeka, Croatia \\ ${ }^{\mathrm{e}}$ Department of Physics, Medical Faculty, University of Rijeka, Rijeka, Croatia \\ ${ }_{\mathrm{f}}$ Department of Histology and Embryology, Medical Faculty, University of Rijeka, Rijeka, Croatia
}

\begin{abstract}
The aim of this study was to investigate the presence of microaggregates of macrophages (CD68 positive cells) in macroscopically normal buccal mucosa in patients with inflammatory bowel disease (IBD). Fifty two patients with clinically and pathohistologically diagnosed IBD, thirty patients with Crohn's disease (CD), twenty two patients with ulcerative colitis (UC), and twenty five controls, matched for sex and age, were involved. The occurrence of CD-68 positive cells microaggregates was more frequent in CD patients comparing with UC patients $(P=0.0093)$, and the controls $(P=0.0001)$ respectively. There was no statistically significant difference in occurrence of CD-68 positive microaggregates in patients with positive microbiological findings $(P=0.8258)$. The results suggest that microaggregates of macrophages are more frequently present in apparently normal buccal mucosa in patients with CD than in patients with UC independently of microbiological findings. Therefore, it could be a potential marker for differentiating patients with Crohn's disease from UC patients, when standard tools failed. (doi: $10.5562 / \mathrm{cca} 1779)$
\end{abstract}

Keywords: CD-68 microaggregates, buccal mucosa, CD, UC, predictive factors

\section{INTRODUCTION}

Inflammatory bowel disease (IBD) is a chronic inflammatory condition of the gastrointestinal tract that manifests as ulcerative colitis (UC) or Crohn's disease (CD). The studies indicate that UC and CD are heterogeneous diseases characterized by various genetic abnormalities that lead to aggressive T-cell responses towards a subset of commensally enteric bacteria. ${ }^{1}$ Furthermore, there is a need to classify patients in relation to phenotypic characteristics of the disease. ${ }^{2}$ So, distinguishing the two conditions (CD and UC) is important because of the similar, but different therapeutic implications (medical and surgical treatment). Differential diagnosis is based upon clinical, radiological, endoscopic and histological criteria but in about $10 \%$ of patients it may be difficult to differentiate Crohn's colitis from UC. ${ }^{3,4}$ Colitis yet to be classified is the new term for the cases where a definitive distinction between UC, $\mathrm{CD}$, or other cause of colitis cannot be established after the history, endoscopy, patohistology and radiology have been taken into account. ${ }^{5}$ Serological markers and the new fecal markers can differentiate active IBD from inactive IBD as well as from irritable bowel syndrome (IBS) ${ }^{6,7}$ Genetics also plays a role, but until now, we have no strict discriminating markers for $\mathrm{CD}$ and $\mathrm{UC}$ as well. So, genetic testing is currently not recommended for routine practice. ${ }^{4}$ Recently, "tissue factor predictors" or mucosal biomarkers are considered as potential predictor in disease progression or response to the therapy. ${ }^{8}$ Microaggregates of immunostained macrophages in noninflammed gastrointestinal mucosa seem to be a useful method for differentiating Crohn's colitis from UC. ${ }^{3,5}$ The aim of this study was to investigate the presence of microaggregates of CD-68 positive cells

\footnotetext{
$\dagger$ Presented at the $10^{\text {th }}$ Congress of the Croatian Society of Biochemistry and Molecular Biology held in Opatija, Croatia, September 15-18, 2010.

* Author to whom correspondence should be addressed. (E-mail: brankica.sincic@medri.hr)
} 
Table 1. Demographic parameters of involved subjects

\begin{tabular}{lllll}
\hline & $\begin{array}{l}\text { IBD } \\
(n=52)\end{array}$ & $\begin{array}{l}\text { Crohn's Disease } \\
(n=30)\end{array}$ & $\begin{array}{l}\text { Ulcerative Colitis } \\
(n=22)\end{array}$ & $\begin{array}{l}\text { Control } \\
(n=25)\end{array}$ \\
\hline Age (years) & & & & \\
Mean \pm s.d. & $40.09 \pm 16.32$ & $36.10 \pm 16.51$ & $45.54 \pm 14.72$ & $35.36 \pm 17.76$ \\
Range & $18-79$ & $18-79$ & $23-74$ & $18-72$ \\
Females : Males & $28: 24$ & $16: 14$ & $12: 10$ & $10: 15$ \\
Remission: Activity & $21: 31$ & $11: 19$ & $10: 12$ & \\
\hline
\end{tabular}

(macrophages) in macroscopically normal buccal mucosa in patients with IBD in order to determinate possible discriminating tissue marker. ${ }^{6}$

\section{EXPERIMENTAL SECTION}

\section{Subjects}

A cross-sectional study involved 52 patients, with a previously clinical and pathohistological diagnosed IBD, 30 with Crohn's disease (CD) and 22 with ulcerative colitis (UC). The patients were in- and outpatients of the Department of Gastroenterology at the Clinical Hospital Centre Rijeka. The control group was constituted of 25 patients who were hospitalized for trauma in maxillofacial region at the Department of Maxillofacial Surgery at the Clinical Hospital Centre Rijeka, and needed surgical treatment in oral cavity. The two groups were matched for sex and age (Table 1). All the subjects were informed about purpose of this study and they freely agreed to be part of it. They all signed an informed consent that was approved by the Hospital Ethics Committee. All of them completed a questionnaire that included medical history and medications used. The diagnosis of CD and UC were established owing to ECCO consensus for UC and CD diagnosis as well. ${ }^{4,5}$ The patients with $\mathrm{CD}$ were divided into active and inactive group based on CDAI (Crohn's Disease Activity Index) classification considering the $\mathrm{CDAI}<150$ as the remission. Patients with UC were classified according to the Montreal classification of severity of the disease. ${ }^{9,10}$ IBD was excluded in control group by history, laboratory values and physical exam.

The gingival and dental health status of patients with IBD and the control group has been investigated.

\section{Histopathology and Immunohistochemistry}

In local anesthesia with $2 \mathrm{~mL}$ of $2 \%$ xylocain, apparently normal (healthy) buccal mucosa in the area of palatal's arches was biopsied. The wound was sewed up with resorptive material. Biopsy specimen's sizes of $0.5-1.0 \mathrm{~cm}$ were fixed in $10 \%$ formalin overnight and embedded in paraffin $\left(50-56^{\circ} \mathrm{C}\right)$. Two serial sections $(3-4 \mu \mathrm{m})$ were cut and deparaffinised in xylene and rehydrated through graded alcohol ( 5 minutes in each). One of them was stained with hematoxylin and eosin (HE) for histopathological screening and the other was prepared for immunohistochemistry.

The sections for immunohistochemistry were washed in distilled water and immersed in ethanol with $3 \%$ hydrogen peroxide to eliminate endogenous peroxidase activity. For antigen retrieval, the slides were immersed in preheated TRIS-EDTA buffer ( $\mathrm{pH} 9)$ on 96-99 ${ }^{\circ} \mathrm{C}$. After 10 minutes, the slides were cooled for new 20 minutes on room temperature and treated with normal mouse serum for 20 minutes to block nonspecific binding. In Dako Techmate Horizon automated immunostainer sections were incubated with monoclonal mouse anti-human CD68 antibody (clone-KP1; M0814) at a dilution of 1:2000. For negative control DakoCytomation Mouse IgG1, code no. X 0931, diluted to the same concentration as the primary antibody was used. Dako Real Detection System, Peroxidase/DAB+, Rabbit/Mouse, was used in immunohistochemistry together with Dako automated immunostaining instruments (code K5001) yelling brown precipitate. For contrast staining hematoxylin was used. After dehydration in 70, 96 and $100 \%$ alcohol, in each for 3 minutes; twice in xylene substitute, slides were dried and mounted with Entelan. Images from an Olympus (BX40) scope were captured and edited with an Olympus digital camera.

Histological Criteria for the Microaggregates of Macrophages

The presence of microaggregates of macrophages at the sections from buccal mucosa was investigated. As aggregate were considered cluster of 5-10 CD-68 positive cells in close contact with each other. The patient was claimed as being positive when at least one microaggregate was found.

\section{Microbiology}

Unstimulated whole saliva samples, and oral mucosa swabs (Copan Venturi Transystem; Copan, Brescia, Italy) were collected in sterile condition and cultured by standard methods. Serial ten-fold dilutions of the saliva samples in sterile phosphate-buffered saline (PBS, pH 7.4) were plated onto different media. The 

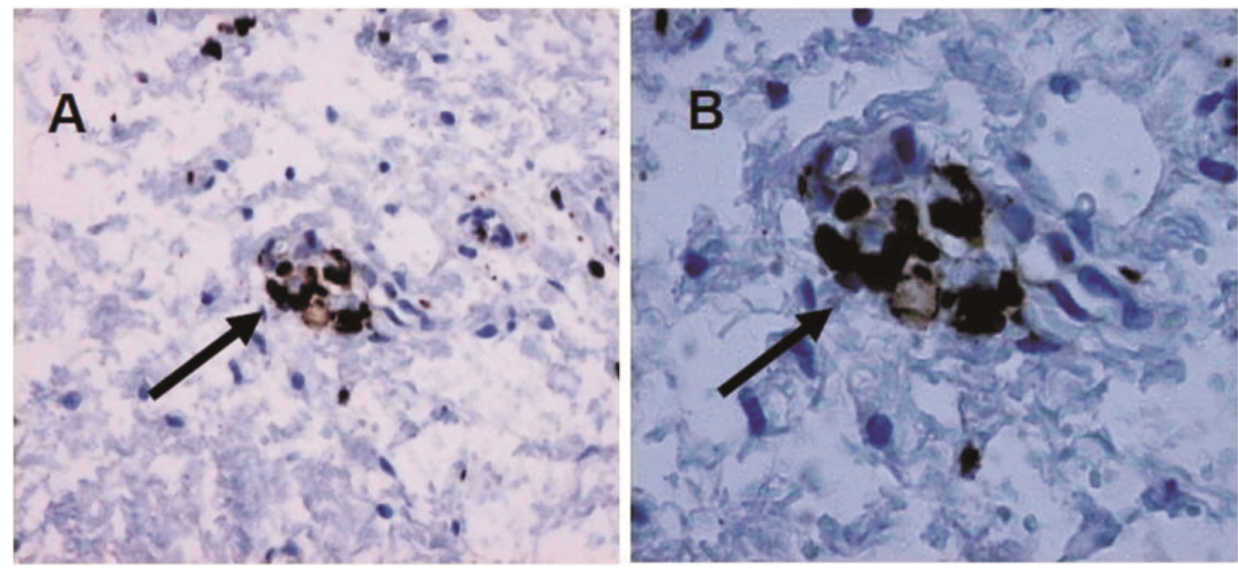

Figure 1. Macrophages in buccal mucosa: Immunohistology of formalin fixed, paraffin embedded buccal mucosa, incubated with monoclonal CD68 antibody. Cluster of CD-68 positive cells (brown staining). Contrastained with hematoxilin. Magnification $200 \times(\mathrm{A}), 400 \times(\mathrm{B})$.

colony-forming units (CFU) of microorganisms on each plate were counted after incubation at $37{ }^{\circ} \mathrm{C} / 48$ $\mathrm{h}$ in ambient air for yeasts, and $37{ }^{\circ} \mathrm{C} / 24 \mathrm{~h}$ in $5 \%$ $\mathrm{CO}_{2}$ atmosphere for bacteria. Chromalbicans Agar (Biolife, Milano, Italy) was used as a selective medium for the isolation and presumptive identification of yeasts. Yeast-like colonies growing on primary isolation medium were tested for germ tube (GT) production and were identified with the API ID 32C (bioMérieux, Marcy l'Etoile, France). GT test was performed according to standard methods. ${ }^{11}$ The API ID $32 \mathrm{C}$ was used according to the manufacturer's instructions. For bacterial cultivation the following media were used: blood agar, MacConkey agar and DifcoTMCetrimide agar (Becton, Dickinson and Company, Franklin Lakes, NJ, USA). A slide agglutination test was used for identification of Staphylococcus aureus (Slidex Staph-Kit; bioMérieux, Marcy 1'Etoile, France). Lancefield serogrouping of betahemolytic streptococci was performed using Slidex StreptoPlus (bioMérieux, Marcy l'Etoile, France). Enterobacteriaceae were identified by the API $20 \mathrm{E}$ test (bioMérieux, Marcy l'Etoile, France).

API 20 NE system (bioMérieux, Marcy l'Etoile, France) was used for identification of nonfermenting gram-negative bacteria.

\section{Statistics}

Statistical analyses were performed as a cross-sectional analysis between patients with UC vs. CD vs. controls. Data are expressed as means $\pm \mathrm{SD}$. Descriptive statistic were done with all data. Fisher's exact, two-tailed test was applied; given the small number of samples within each group. The level of $P<0.05$ was defined as statistically significant. STATISTICA version 8.0 was used for all calculations.

\section{RESULTS}

Fifty-two IBD patients and 25 controls were studied. Sex, age, and disease duration and activity distribution are presented in Table 1. Pathohistological findings of CD-68 positive cells microaggregates (immunostained macrophages) in buccal mucosa are shown in Figure 1 . Microaggregates were found in $17(57 \%)$ patients with $\mathrm{CD}$ and in $4(18 \%)$ patients with UC. There were three positive control patients for the respective findings. The occurrence of CD-68 positive cells was more frequent in CD patients comparing with UC patients $(P=0.0093)$, and the controls $(P=0.0001)$ respectively. There was no difference $(P=0.7423)$ between UC patients and the control group regarding the occurrence of the same microaggregates (Figure 2). The sensitivity of CD-68 microaggregate finding in buccal mucosa for CD diagnosis was $57 \%$, with high specificity of $82 \%$. There were no statistical significance in appearances of this findings between male and female with $\operatorname{IBD}(P=0.1621)$, neither with $\mathrm{CD}(P=0.7131)$ nor with UC $(P=0.0963)$. Furthermore, comparing macrophage microaggregate in buccal mucosa in the patients with active $\mathrm{CD}$ and patients in remission, no statistical significance was detected $(P=$ 0.5567) (Figure 3). The occurrence of CD-68 microaggregate was not more frequent in $\mathrm{CD}$ patients with upper gastrointestinal involvement $(P=0.348)$. Also, it didn't depend on the duration of the disease $(P=$ 0.8193 ). Furthermore, there was no statistical significance in appearance of Candida albicans in saliva or in smear of oral cavity neither between patients with IBD and control group $(P=0.8165)$, nor between $\mathrm{CD}$ and UC patients $(P=0.6637)$. Also, there was no statistically significant difference in occurrence of CD-68 positive microaggregates in patients with positive microbiological findings $(P=0.8258)$. The presence of aphthous lesion 


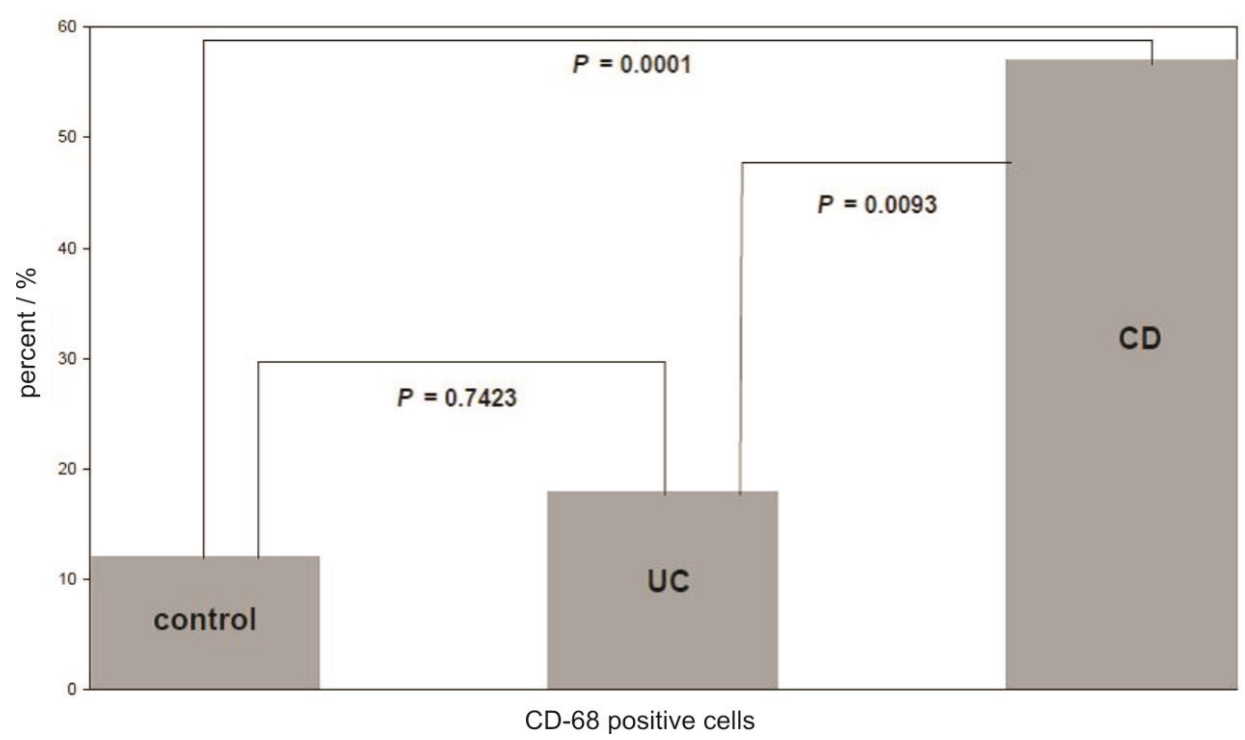

Figure 2. CD68-positive cells in sections of buccal mucosa: Comparisons between patients with Crohn's disease (CD; $n=17 / 30)$, ulcerative colitis (UC; $n=4 / 22$ ), Inflammatory bowel disease (IBD; $n=21 / 52$ ) and control individuals (CTRL; $n=3 / 25$ ).

was not connected with more frequent occurrence of CD68 positive microaggregate $(P=0.5579)$.

\section{DISCUSSION}

Nowadays, distinguishing not only CD from UC, but also different phenotypes of $\mathrm{CD}$ and $\mathrm{UC}$ represents an imperative of clinical approach to inflammatory bowel disease treatment, and it would be ideal to have validated prognostic markers as a tool for predicting future behaviour of disease. So, recognition of importance in finding typical entities has induced the large searching among clinicians as well as laboratory workers. ${ }^{8,12-14}$ In our previous study we investigate clinical relevance of the serum enzyme dipeptidyl peptidase IV (DPP IV) activity in patients with inflammatory bowel disease and showed that it couldn't be the discriminating marker among $\mathrm{CD}$ and $\mathrm{UC} .^{15}$ In accordance with the results of the present study, microaggregates of $\mathrm{CD}-68$ positive cells in macroscopic healthy buccal mucosa could be helpful marker that can make difference between Crohn's disease and ulcerative colitis patients. Crohn's disease can affect any part of the gut, and lesions can be found in the oral cavity as disease manifestation or as disease related lesions. ${ }^{16}$ Oral lesions have been de-

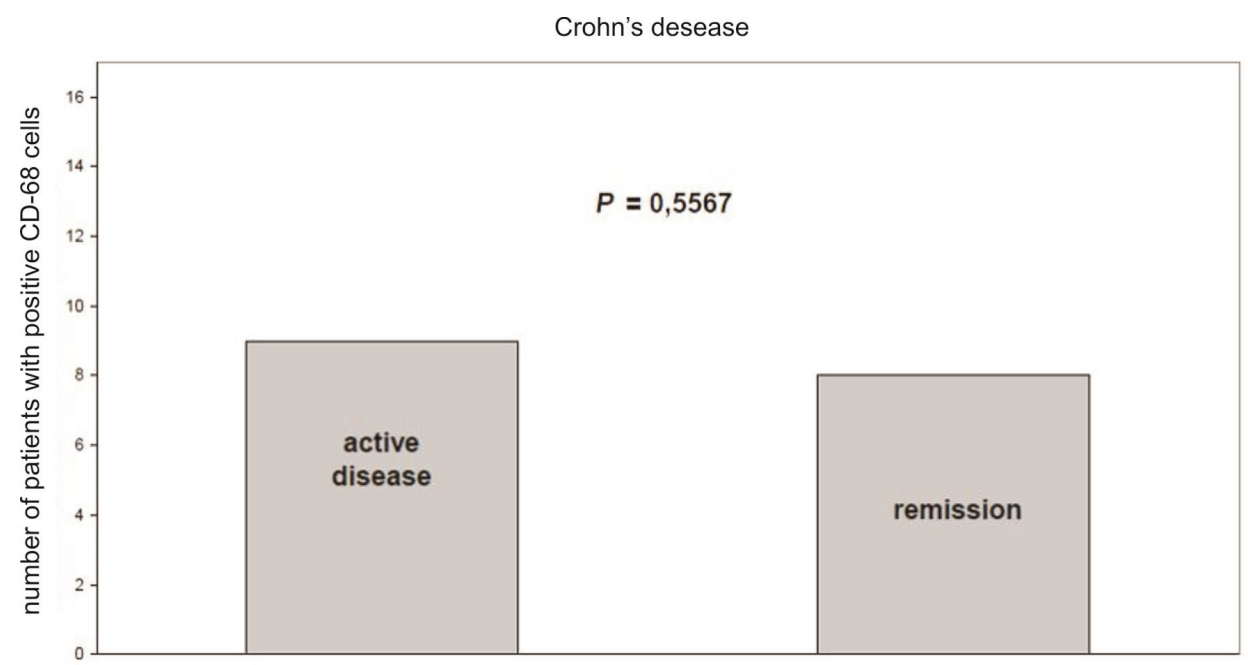

Figure 3. CD68-positive cells in sections of buccal mucosa in patients with Crohn' disease: Microaggregates were found in 17 patients with $\mathrm{CD}(n=30)$. Among patients there were nine with active disease and eight in remission. No statistical significance was detected comparing macrophage microaggregate in buccal mucosa in the patients with active CD and patients in remission $(P=0.5567)$. 
scribed more frequent in Crohn's disease than in ulcerative colitis. The lesions may precede or accompany gastrointestinal disease and can be the only site of involvement. In Crohn's disease there are aphtoid ulcerations, granulomatous autoimmune-like changes in minor salivary glands, pyostomatitis vegetans, dental erosion, candidiasis and a high prevalence of caries. In ulcerative colitis patients oral lesions may include mucosal ulcers, pyostomatitis vegentans, diffuse pustules and lichen planus. ${ }^{16-20}$ Patients with active Crohn's disease have more oral signs compared with non-active Crohn's disease. ${ }^{17}$ All those findings were specified in macroscopic changed mucosa, but microaaggregates of CD-68 positive cells, in healthy oral mucosa of IBD patients have not yet been described.

Furthermore, human oral cavity contains more than 500 bacterial species inhabiting the teeth, gingival crevices, buccal mucosa and tongue. ${ }^{21}$ Clusters of CD68 positive cells were found in some infective diseases; in Borrelia burgdorferi and Eikenella corrodens infections, but also in intestinal Behcet's disease or some inflammatory tumor associated with infection. ${ }^{22-25}$ In present study sporadic findings of enterobacteriaceae, streptococci and staphylococci were not statistically different in patients with IBD comparing to the controls. Also, there was no statistical significance in appearance of Candida albicans in saliva or in smear of oral cavity neither between patients with IBD and control group, nor between $\mathrm{CD}$ and UC patients. There was no statistically significant difference in occurrence of CD-68 positive microaggregates in patients with positive microbiological findings, so it could be excluded as the possible reason of microaggregates. Yao and coworkers detected microaggregates of immunostained macrophages in a biopsy specimen taken from noninflamed gastroduodenal mucosa only in patients with Crohn's colitis, but not in ulcerative colitis. ${ }^{3}$ So, it was described as a useful histological marker in differentiating Crohn's colitis from ulcerative colitis. As the results of the present study in oral mucosa show high specificity of macrophages microaggegates for Crohn's disease it could also be a very simple tool in differentiating ulcerative colitis from Crohn's colitis. Additionally, the appearances of microaggregates of macrophages in buccal mucosa were not in a correlation neither with upper gastrointestinal involvement, nor disease activity and it could be used at any time of the disease course. So it could be helpful in selecting the appropriate medical and surgical treatment for the individual patients. Ileal pouch-anal anastomosis (IPAA) is the most recently devised procedure for ulcerative colitis and avoids the need for permanent ileostomy. On the contrary, evidence suggests that in patients with Crohn's disease complication and failure rates are higher after IPAA. At present, an IPAA is not recommended in a patient with Crohn's disease. ${ }^{26,27}$
In conclusion, microaggregates of CD-68 positive cells in macroscopic healthy buccal mucosa could be helpful marker which can contribute to differentiation between Crohn's colitis and UC patients. Further studies regarding microaggreagates of $\mathrm{CD}-68$ positive cells, as a potential disease predictor, are necessary.

Acknowledgements. We thank Marina Bubonja for her work in microbiology part of the manuscript. This work was supported by Croatian Ministry of Science, Education and Sport grant 062-0000000-0219.

\section{REFERENCES}

1. R. B. Sartor, Nat Clin Pract Gastroenterol Hepatol 3 (2006) 390-407.

2. J. Cosnes, S. Cattan, A. Blain, L. Beaugerie, F. Carbonnel, R. Parc, and J. P. Gendre, Inflamm Bowel Dis 8 (2002) 244-250.

3. K. Yao, T. Yao, A. Iwashita, T. Matsui, and S. Kamachi, Am J Gastroenterol 95 (2000) 1967-1973.

4. A. Van Assche, J. Panes, L. Beaugerie, J. Karagiannis, M. Allesz, T. Ochsenkuhn, T. Orchard, G. Rogler, E. Louis, L. Kupcinskas, G. Mantzaris, S. Travis, and E. Stange, J Crohn's Colitis 4 (2010) 7-27.

5. S. P. Stange, S. Vermeire, W. Reinisch, K.Geboes, A. Barakaauskiene, R. Feakis, J. F. Flejou, H. Herfarth, D. W. Hommes, L. Kupinsakas, P. L. Lakatos, G. J. Mantzaris, S. Schreiber, V. Villanacci, and B. F. Warren, J Crohn's Colitis 2 (2008) 1-23.

6. J. Langhorst, S. Elsenbruch, J. Koelzer, A. Rueffer, A. Michalsen, and G. J. Dobos, Am J Gastroenterol 103 (2008) 162-169.

7. L. Peyrin-Biroulet, A. Standaert-Vitse, J. Branche, and M. Chamaillard, Inflamm Bowel Dis 13 (2007) 1561-1566.

8. F. Scaldaferri, C. Correale, A. Gasbarrini, and S. Danese, World $J$ Gastroenterol 16 (2010) 2616-2625.

9. W. R. Best, J. M. Becktel, and J. W. Singleton, Gastroenterology 77 (1979) 843-846.

10. M. S. Silverberg, J. Satsangi, T. Ahmad, I. D. Arnott, C. N. Bernstein, S. R. Brant, R. Caprilli, J. F. Colombel, C. Gasche, K. Geboes, D. P. Jewell, A. Karban, E. V. Loftus Jr, A. S. Pena, R. H. Riddell, D. B. Sachar, S. Schreiber, A. H. Steinhart, S. R. Targan, S. Vermeire, and B. F. Warren, Can J Gastroenterol 19 Suppl A (2005) 5-36.

11. D. H. Larone, Medically important fungi, a guide to identification, $3^{\text {rd }}$ ed., Washington DC, 1995.

12. C. Loly, J. Belaiche, and E. Louis, Scand J Gastroenterol 43 (2008) 948-954.

13. M. J. Romberg-Camps, P. C. Dagnelie, A. D. Kester, M. A. Hesselink-van de Kruijs, M. Cilissen; L. G. Engels, C. Van Deursen, W. H. Hameeteman, F. L. Wolters, M. G. Russel, and R. W. Stockbrugger, Am J Gastroenterol 104 (2009) 371-383.

14. B. G. Feagan, R. Panaccione, W. J. Sandborn, G. R. D'Haens, S. Schreiber, P. J. Rutgeerts, E. V. Loftus Jr., K. G. Lomax, A. P. $\mathrm{Yu}$, E. Q. Wu, J. Chao, and P. Mulani, Gastroenterology 135 (2008) 1493-1499.

15. J. Varljen, L. Batičić, N. Varljen, D. Detel, and A. Lekić, Croatica Chemica Acta 78 (2005) 427-432.

16. E. A. Field and R. B. Allan, Aliment Pharmacol Ther 18 (2003) 949-962.

17. G. Bens, D. Laharie, M. Beylot-Barry, B. Vergier, I. Noblesse, C. Beylot, and M. Amouretti, Br J Dermatol 149 (2003) 181-184.

18. H. J. Scheper and H. S. Brand, Int Dent J 52 (2002) 163-172. 
19. J. Katz, A. Shenkman, F. Stavropoulos, and E. Melzer, Oral Dis 9 (2003) 34-40.

20. S. Basu, J Indian Med Assoc 64 (1975) 327-329.

21. H. Tlaskalova-Hogenova, R. Stepankova, T. Hudcovic, L. Tuckova, B. Cukrowska, R. Lodinova-Zadnikova, H. Kozakova, P. Rossmann, J. Bartova, D. Sokol, D. P. Funda, D. Borovska, Z. Rehakova, J. Sinkora, J. Hofman, P. Drastich, and A. Kokesova, Immunol Lett 93 (2004) 97-108.

22. A. B. Farris $3^{\text {rd }}$ and R. L. Kradin, Virchows Arch 449 (2006) 726-729.

23. K. Nara, M. S. Kurokawa, S. Chiba, H. Yoshikawa, S. Tsukikawa, T. Matsuda, and N. Suzuki, Clin Exp Immunol 152
(2008) 245-251

24. K. J. Lee, M. Inoue, T. Otani, M. Iwasaki, S. Sasazuki, and S. Tsugane, Int J Cancer 118 (2006) 2315-2321.

25. A. Fernandez-Flores and E. Ruzic-Sabljic, Acta Dermatovenerol Alp Panonica Adriat 17 (2008) 171-176.

26. J. M. Hwang and M. G. Varma, World J Gastroenterol 14 (2008) 2678-2690.

27. S. P. Travis, E. F. Stange, M. Lemann, T. Oresland, Y. Chowers, A. Forbes, G. D'Haens, G. Kitis, A. Cortot, C. Prantera, P. Marteau, J. F. Colombel, P. Gionchetti, Y. Bouhnik, E. Tiret, J. Kroesen, M. Starlinger, and N. J. Mortensen, Gut 55 Suppl 1 (2006) i16-i35. 